\title{
Resonant Soft-x-ray Emission Spectroscopy of Liquids
}

\author{
J.-H. Guo ${ }^{\dagger}$, A. Augustsson ${ }^{\dagger}$, C.-J. Englund", and J. Nordgren ${ }^{\ddagger}$ \\ †Advanced Light Source, Lawrence Berkeley National Laboratory, Berkeley, CA 74720 \\ IDepartment of Physics, Uppsala University, Box 530, S-751 21 Uppsala, Sweden
}

\begin{abstract}
We present now a possible way to carry out soft-x-ray fluorescence spectroscopy of liquids. The liquid cell has a window to attain compatibility with UHV conditions of the spectrometer and beamline, The synchrotron radiation enters the liquid cell through a $100 \mathrm{~nm}$-thick silicon nitride window and the emitted xrays exit through the same window. This allows in particular liquid-solid interfaces to be studied. Such a liquid cell has been used to study the electronic structure of a variety of systems ranging from water solutions of inorganic salts and inertial drugs to nano materials and actinide compounds in their wet conditions.
\end{abstract}

\section{Introduction}

So far the electronic structure and dynamics in biological systems has been little investigated. Synchrotron based x-ray diffraction is used to determine the molecular geometry that in many cases can determine the biological function of the molecule. The electronic structure determines the properties of matter, and it is therefore natural to anticipate that a description of the electronic structure of biological systems will lead to a progress in molecular biology. When studying biological systems it is necessary to be able to perform the experiments in their natural environment, i.e. in water/liquid solutions.

A very important but hitherto less addressed question is that of the hydrogen bond effect on the electronic structure. This effect is essential for understanding the physical and chemical properties of many chemical and biological systems. The reason for this neglect, however, is the limited experimental access to the electronic structure of liquids. The application of spectroscopic methods to study the electronic structure of liquids has been hampered by the incompatibility of wet samples and high-vacuum conditions. Hence, even understanding the properties of pure liquid water remains a challenge.

Soft-x-ray emission spectroscopy is essentially bulk sensitive, since the attenuation length of photons in this energy range is typically hundreds of nanometers in solid matter. The penetration depth offers a few experimental opportunities not present in electron based spectroscopy. We have taken advantage of the large photon attenuation length to perform the first soft-x-ray emission study of liquid water and solutions [1]. Most liquids have high vapor pressure (such as water: 20 Torr of partial pressure at room temperature). We can't just simply stick the liquid on a sample plate and transferee it into the vacuum chamber. For this purpose we constructed a liquid cell for soft-x-ray emission spectroscopic studies of liquids.

\section{Experimental Set-Up}

The experiments were performed at beamline 7.0.1 at the Advanced Light Source [2]. A thin window (100 $\mathrm{nm}$ silicon nitride), separating the liquid from the surrounding vacuum, is penetrated both by the incident photon beam and the secondary x-ray emission. A high resolution grating spectrometer [3] was mounted parallel to the polarization vector of the incident photon beam to detect the emitted $\mathrm{x}$-ray. X-ray absorption spectra of liquids were also measured in fluorescence yield mode using the same setup. The grazingincidence grating spectrometer is based on Rowland geometry. It consists of slit, gratings and detector. Three spherical gratings are optimized to cover an operation range of 50-1000 eV. The gratings are mounted at angles of incidence to have a joint fixed slit, and the emitted x-rays are detected using a two-

CP705, Synchrotron Radiation Instrumentation: Eighth International Conference, edited by T. Warwick et al.

(C) 2004 American Institute of Physics 0-7354-0180-2/04/\$22.00

1066 
dimensional detector that can be positioned and oriented tangentially to the pertained Rowland circle. The detector consists of multi channel plates and a resistive anode with a four-electrode readout.

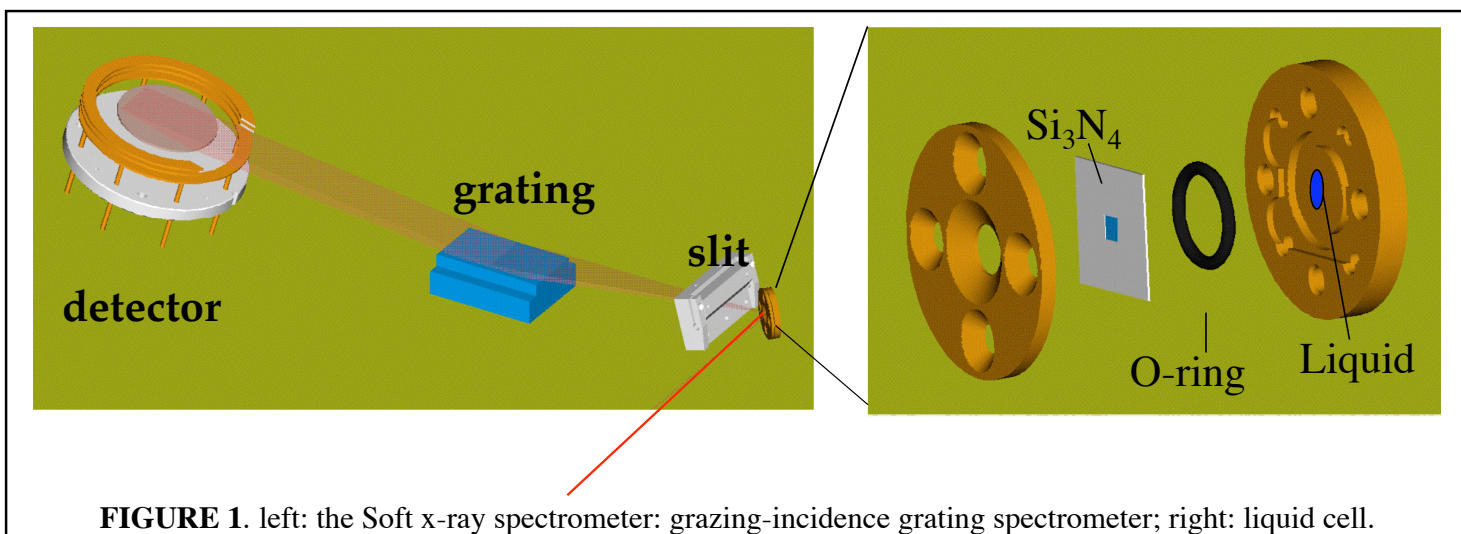

The liquid cell has a window to attain compatibility with UHV conditions of the spectrometer and beamline, The synchrotron radiation enters the liquid cell through a $100 \mathrm{~nm}$ thick silicon nitride window and the emitted $\mathrm{x}$-rays exit through the same window. The thin silicon nitride window is commercial available [4]. The test showed that $100 \mathrm{~nm}$ thin $\mathrm{Si}_{3} \mathrm{~N}_{4}$ membrane of $2.25 \times 2.25 \mathrm{~mm}^{2}$ could hold one atmosphere pressure. Our liquid cell (see in Figure 1) consists of a metal container and $1 \times 1 \mathrm{~mm}^{2}$ and 100 $\mathrm{nm}$ thin $\mathrm{Si}_{3} \mathrm{~N}_{4}$ membrane, which can withstand the differential pressure between the liquid on one side and UHV on another side. The incoming and outgoing photons are traveling through the same window. The transmission of x-rays at the $\mathrm{C}, \mathrm{O} K$-edge, and $\mathrm{Fe} L$-edge for a $100 \mathrm{~nm}$ thick window is about $46 \%, 66 \%$, and $82 \%$, respectively (see in Figure 2). Silicon nitride membrane is not the best choice comparing to other materials with respect to its transmission of $\mathrm{x}$-ray in the energy region of $80-1000 \mathrm{eV}$, but its mechanical properties and fabrication process make it only candidate at present stage. The experiments have been done at the vacuum pressure of less than $1 \times 10^{-9}$ Torr.

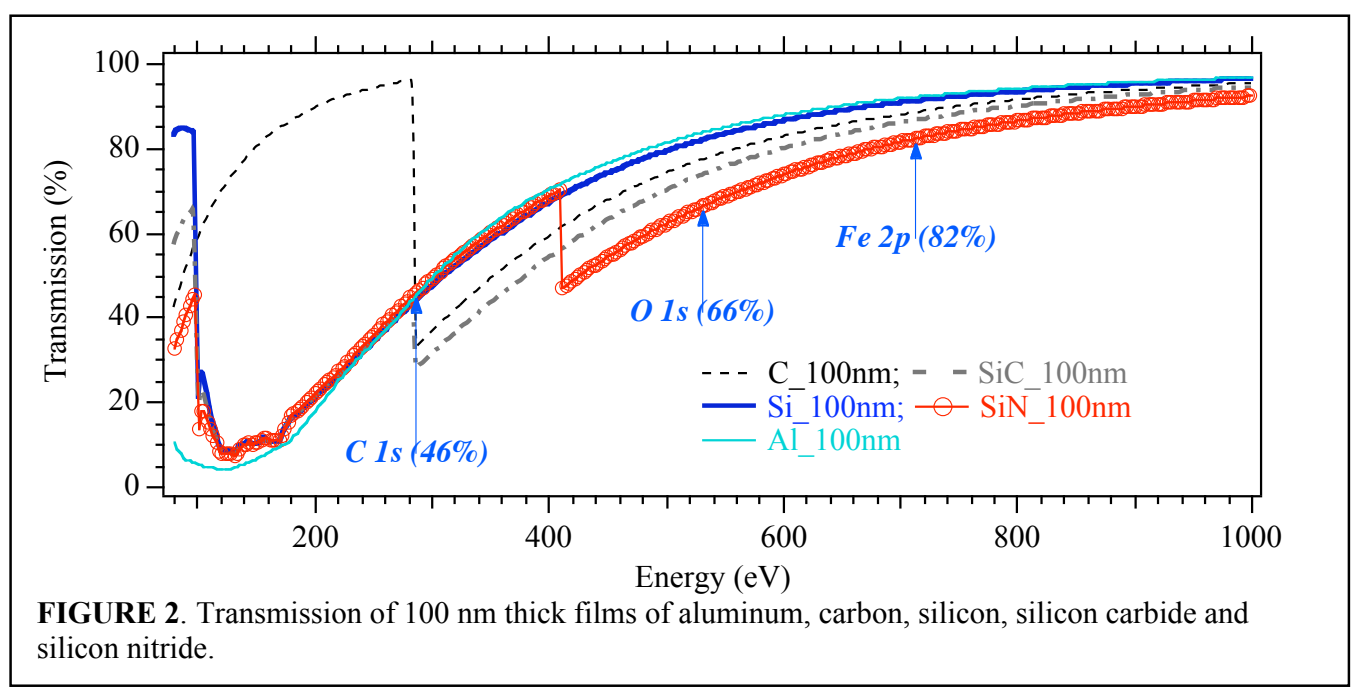

\section{Applications}

Selectively excited soft-x-ray emission spectroscopy provides a unique approach to determine the properties of liquids and solutions. Pauling stated in the 50's that electron sharing between water molecules results in a covalency in the hydrogen bond. Many attempts have been made in the past to verify Pauling's prediction, but without much success due to the limitation of experimental access to the electronic structure of liquids. Figure 3 reports the first soft-x-ray emission spectra of liquid water. Soft-x-ray emission is a direct probe of the local electronic structure of complex systems. Our experimental and theoretical studies 
on liquid water provide clear evidence that an electron sharing takes place between water molecules. Such a sharing mainly involves the so-called $3 \mathrm{a}_{1}$ orbital, which is a mixing of oxygen $2 p$ and hydrogen $2 s$ atomic orbitals. The outermost "lone pair" orbital $\left(1 b_{1}\right)$, however, hardly shows any change upon solvation, which is in contradiction with the normal definition of so-called coordinate-covalent bonding (also called donoracceptor or Lewis acid-base bonding). Moreover, the soft-x-ray emission spectra of liquid water nicely show the origin for the increasing of dipole moment in liquid water, and they have also been used to separately determine a particular structure with broken hydrogen bonding.
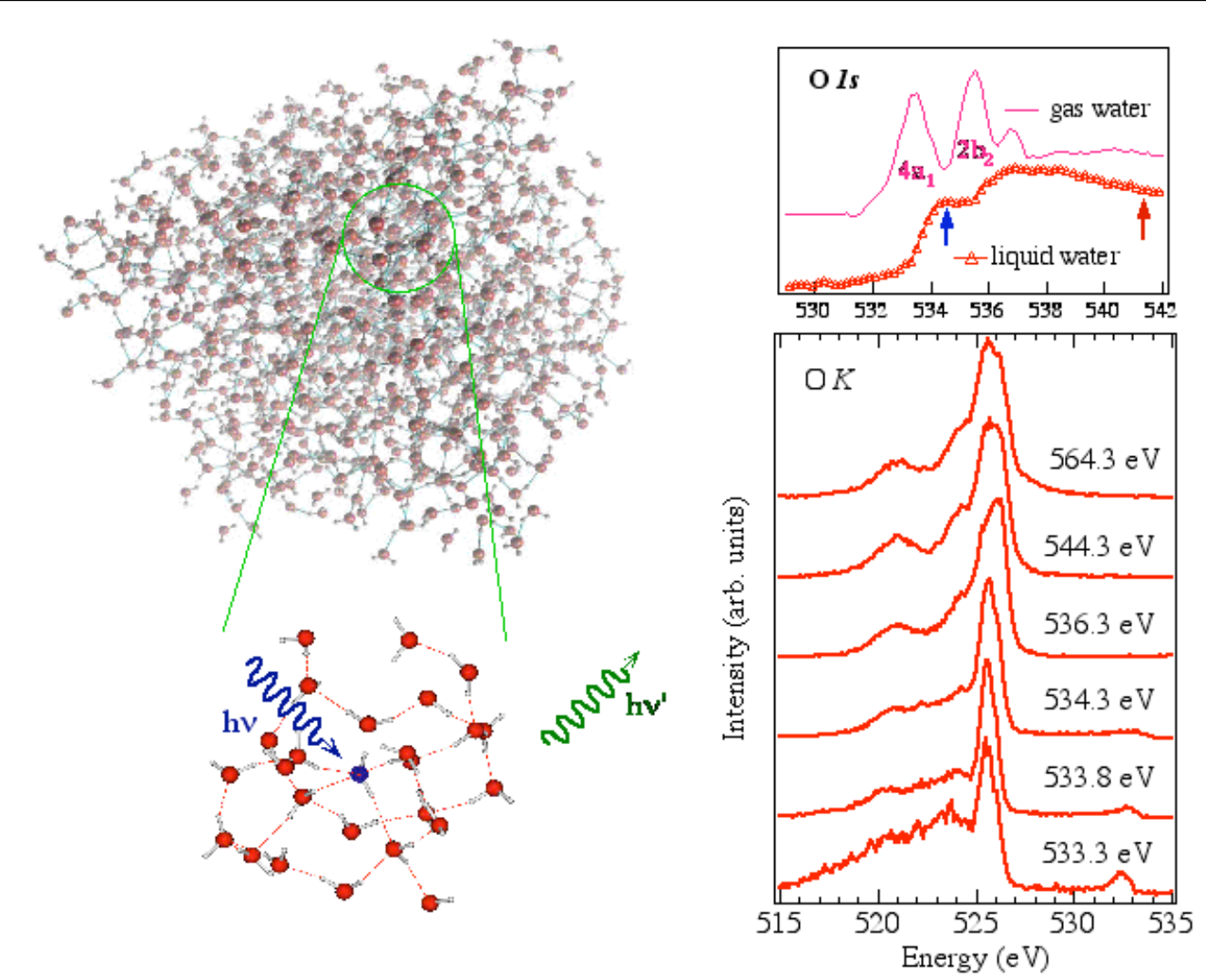

FIGURE 3. X-ray emission spectra of the water molecules and liquid water, formed as electrons from the three outermost occupied molecular orbitals, depicted in the right panel, fill a vacancy in the $1 \mathrm{a}_{1}$ core level.

Another recent study of selectively excited soft-x-ray emission spectroscopy on the mixture of liquid water and alcohol provides information about the molecular arrangements [5]. We find that liquid methanol is a combination of 6- and 8-rings and 6- and 8-chains, and we learn that the solute-solvent interaction takes place mainly between chains and water molecules. In this manner we have substantially refined both the knowledge of structure and order present in a methanol-water solution and the understanding of the unusual thermodynamic properties. The understanding of pure liquid methanol has been improved as well. The fact that the local electronic structure is so sensitive to the molecular arrangements demonstrates the complexity of solutions.

Metal-ion transport in both aqueous- and polymer-solvent media involves continual solvent-ligand exchange. Metal-ion coordination chemistry is therefore fundamental to these phenomena where a dramatic exchange of ligand occurs. Optical, infrared and Raman spectroscopies as well as magnetic resonance based methods are often used to determine the structure and the properties of these systems. In Figure 4, we report the soft-x-ray absorption and emission study of cations $\left(\mathrm{Na}^{+}, \mathrm{Mg}^{2+}\right.$, and $\left.\mathrm{Al}^{3+}\right)$ in water solutions.

Alternatively the effects of solvated cations could be monitored via examining the spectra of solvent. The great advantage of this approach is that the restriction to cations no longer applies. For example, it can be used in studying what happens when salts of the alkali and alkaline earth metals are added to aqueous solvent. This study made it possible to establish a relative order cation-solvent (water) interaction.

Figure 4 shows the X-ray absorption and emission studies of liquid water and $\mathrm{NaCl}, \mathrm{MgCl}_{2}$, and $\mathrm{AlCl}_{3}$ 
water solutions. The influences of cations on the water molecular structure can be seen as the threshold shifted towards high energy in the x-ray absorption spectra (Figure 4a); the mixing of molecular orbital in $3 \mathrm{a}_{1}$ symmetry is reinforced as the intensity of $3 \mathrm{a}_{1}$ is further reduced (indicated by arrow in Figure $4 \mathrm{~b}$ ); the $1 b_{1}$-emission peak is shifted differently for $\mathrm{Na}^{+}, \mathrm{Mg}^{2+}$, and $\mathrm{Al}^{3+}$, which indicates that the charge difference of the cations may not be the only playing role being responsible to the interactions between the cations and water molecules.

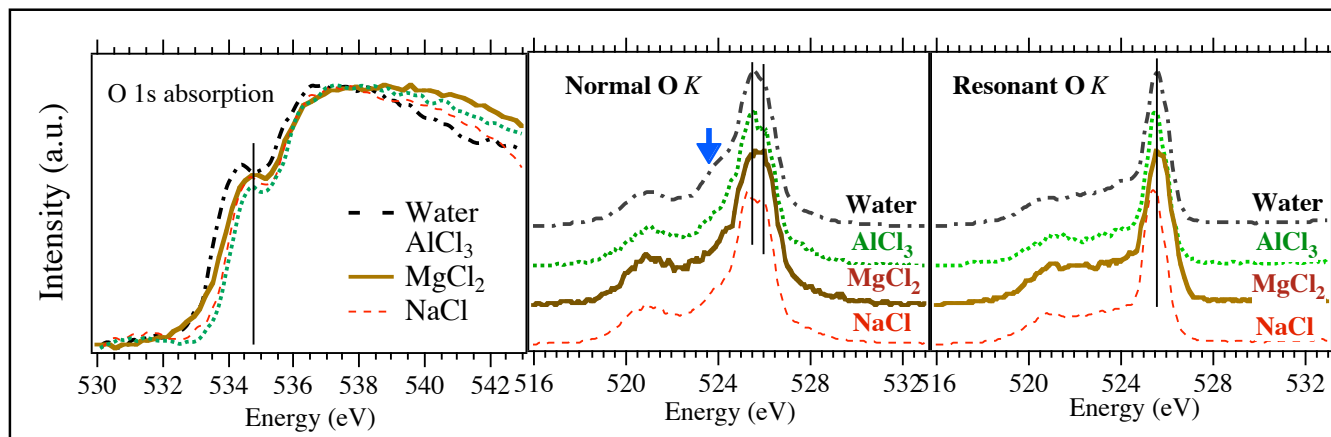

FIGURE 4. X-ray absorption and selectively excited x-ray emission spectra of liquid water and $\mathrm{NaCl}$, $\mathrm{MgCl}_{2}$, and $\mathrm{AlCl}_{3}$ water solutions.

A suspension liquid of Co metal nanoparticles has been studied [6]. Surface oxidation was avoided in our studies of 9-nm Co nanoparticles by passivating the NCs' surfaces with oleic acid during synthesis under air- and water-free conditions. Additionally, the sample was kept as a liquid suspension of nanoparticles in anhydrous and air-free 1,2 dichlorobenzene, which provided protection from oxidation.

The liquid cell was also used in the spectroscopic analysis of several samples relevant to the processes governing the behavior of oxidized uranium species in groundwater solutions under anoxic conditions [7]. Both Fe samples with different times of exposure to the U(IV) solution and uranium species in the solution in the liquid cell with Fe were studied. The specific spectroscopic technique used in current study is based on resonant inelastic soft-x-ray scattering, which has been shown to be sensitive to the chemical state of uranium. The measurements were performed at a number of energies of the primary photon beam across the $\mathrm{U} 5 d$ absorption edge. The results unambiguously indicate the reduction of U(VI) to U(IV) on the Fe surface.

\section{Acknowledgements}

We thank S. Butorin, L. Duda, and J.-E. Rubensson for stimulating discussion in design of the liquid cell. The work is supported by the U.S. Department of Energy, under Contract No. DE-AC03-76SF00098 and by the Swedish Research Council.

\section{References}

1. J.-H. Guo, Y. Luo, A. Augustsson, J.-E. Rubensson, C. Såthe, H. Ågren, H. Siegbahn, and J. Nordgren, Phys. Rev. Lett. 89, 137402 (2002).

2. T. Warwick, P. Heimann, D. Mossessian, et al., Rev. Sci. Instr. 66, 2037-2040 (1995).

3. J. Nordgren, G. Bray, S. Cramm, R. Nyholm, et al., Rev. Sci. Instr. 60, 1690-1695 (1989).

4. Silson Ltd, JBJ Business Park, Northampton Road, Blisworth, Northampton, NN7 3DW, England. (E-mail: peter.anastasi@silson.com)

5. J.-H. Guo, Y. Luo, A. Augustsson, S. Kashtanov, J.-E. Rubensson, D. Shuh, H. Ågren, and J. Nordgren, Phys. Rev. Lett. 91, 157401 (2003).

6. F.G. Requejo, G. Thornton, A. Augustsson, J.-H. Guo, D.M. Aruguete, P. Alivisatos, and M. Salmeron, to be published (2003).

7. S. M. Butorin, D. K. Shuh, K. Kvashnina, I. L. Soroka, K. Ollila, K. E. Roberts, J.-H. Guo, L. Werme, and J. Nordgren, to be published (2003). 